\title{
Salvage chemotherapy of biweekly irinotecan plus S-1 (biweekly IRIS) in previously treated patients with advanced gastric cancer
}

\author{
Hong Jae Chon · Sun Young Rha $\cdot$ Hyung Soon Park • \\ Sang Joon Shin • Hyo Song Kim • Jae Kyung Roh • \\ Sung Hoon Noh • Hyun Cheol Chung • Hei-Cheul Jeung
}

Received: 10 October 2010/ Accepted: 16 January 2011 / Published online: 16 February 2011

(C) The Author(s) 2011. This article is published with open access at Springerlink.com

\begin{abstract}
Purpose This phase II trial first describes the combination chemotherapy of biweekly irinotecan plus S-1 (biweekly IRIS) for pretreated advanced gastric cancer (AGC) patients. Methods Patients who had previously been treated with greater than or equal to one regimen were enrolled. They received S-1 $35 \mathrm{mg} / \mathrm{m}^{2}$ twice daily on days $1-14$ and irinotecan $150 \mathrm{mg} / \mathrm{m}^{2}$ on days 1 and 15 , every 4 weeks. The primary endpoint was overall survival (OS).
\end{abstract}

H. J. Chon · S. Y. Rha $\cdot$ S. J. Shin $\cdot$ H. S. Kim .

J. K. Roh · H. C. Chung

Division of Medical Oncology, Yonsei Cancer Center,

Yonsei University College of Medicine, Seoul, Korea

H. S. Park · H.-C. Jeung

Division of Oncology, Gangnam Severance Hospital,

Yonsei University College of Medicine, Seoul, Korea

H. J. Chon - S. Y. Rha - H. S. Park - S. J. Shin ·

H. S. Kim · J. K. Roh · H. C. Chung · H.-C. Jeung

Department of Internal Medicine,

Yonsei University College of Medicine, Seoul, Korea

S. Y. Rha - S. J. Shin $\cdot$ H. S. Kim · J. K. Roh ·

H. C. Chung · H.-C. Jeung

Cancer Metastasis Research Center,

Yonsei University College of Medicine, Seoul, Korea

S. H. Noh

Department of Surgery, Yonsei University College

of Medicine, Seoul, Korea

H.-C. Jeung $(\bowtie)$

Division of Oncology, Department of Internal Medicine, Gangnam Severance Hospital, Yonsei Cancer Center, Yonsei University College of Medicine,

712, Eonju-Ro (146-92, Dogok-Dong), Gangnam-Gu, Seoul 135-720, Korea

e-mail: jeunghc1123@yuhs.ac
Results Among the 38 patients enrolled, 18 patients were treated as second line, and the remaining 20 patients were enrolled as third- or fourth line. A total of 208 cycles were administered with the median being four cycles (range 1-16). The median OS was 8.7 months [95\% confidence interval (CI) 7.5-10.3], and the median progression-free survival was 6.3 months (95\% CI 5.3-7.3). Low serum albumin $(<3.5 \mathrm{mg} / \mathrm{dL})$ was an independent adverse prognosticator for survival. Overall response rate was $17 \%$ (95\% CI 4-30\%). The major grade 3/4 toxicities were neutropenia (26\%) and diarrhea (18\%).

Conclusions Biweekly IRIS showed the moderate activity as salvage treatment in AGC. Considering high neutropenia and gastrointestinal toxicity, patient selection should be warranted; serum albumin may be a predictive factor for treatment decision.

Keywords Gastric cancer - Chemotherapy ·

Clinical trial $\cdot \mathrm{S}-1 \cdot$ Irinotecan

\section{Introduction}

Although gastric cancer is considered chemosensitive, the prognosis is still poor and prolonging survival and improving the quality of life are challenges for oncologists. Combining chemotherapies is the standard approach in metastatic or recurrent gastric cancer, but the overall response rate (ORR) of first-line chemotherapy has been only $35-45 \%$, with a median progression-free survival (PFS) of 5-6 months [1]. This means that many patients eventually develop progressive disease (PD) leading to treatment cessation. Recent advances of chemotherapy have enabled many patients to maintain good performance status even after first-line chemotherapy. In Asian 
countries, including Korea, salvage chemotherapy is widely accepted after first-line treatment fails assuming that it might be helpful for survival prolongation, but little is known about the indication, outcomes, and predictive factors for salvage chemotherapy [2]. Recently, some trials_-mainly phase II studies_- have been conducted for this clinical setting. Various chemotherapy regimens have been tried; cisplatin combinations have shown ORR of $19-45 \%$ [3-5]; paclitaxel combinations have shown ORR of 22-27\% [6, 7]; and irinotecan-based regimens have shown ORR of $12-31 \%$ [8-10]. However, tumor responses and their durations were heterogenous, depending on treatment regimens, response and composition of previous chemotherapy, and patient selected. Moreover, considerable toxicity accompanied many of these regimens. Therefore, prolonging survival with maintaining general performance should be a goal of salvage chemotherapy.

$\mathrm{S}-1$ is one of the active agents currently available in gastric cancer. Encouraging results from several studies evaluating the efficacy of combination partners with S-1 have been reported. Among these, irinotecan is the only agent proving that second-line chemotherapy prolongs survival when compared with best supportive care [11]. Although the study is insufficient - too small patients (only 40 patients) were randomized due to poor accrual to provide convincing evidence of benefit of salvage chemotherapy-irinotecan could be a reasonable partner for combination with S-1, preclinically and clinically. Irinotecan combined with 5-fluorouracil (5-FU) has proven efficacy in colorectal cancer and is also supposed to be active against gastric cancer. Recently, a systematic review suggested irinotecan/5-FU be an appropriate alternative to 5-FU/cisplatin, with a moderate survival benefit and more favorable toxicity profiles [12]. In vitro synergism, different action and toxicity mechanisms, and a lack of cross-resistance also justify combining these two agents. A preclinical study showed that combining SN-38 followed by $5-\mathrm{FU}$ inhibited thymidylate synthase (TS) for a longer period and increased 5-FU metabolite integration into DNA [13]. Moreover, irinotecan reduces TS gene expression, and combining these agents has potent antitumor activity in 5-FU resistant cells [14]. Based on these findings, we conducted the first clinical study to investigate the feasibility of these combinations as salvage treatment after failure of previous chemotherapy.

\section{Patients and methods}

\section{Eligibility}

Patients with histologically confirmed recurrent or metastatic gastric adenocarcinoma were considered eligible when they met all the following criteria: (1) age $\geq 18$ years; (2) Eastern Cooperative Oncology Group (ECOG) performance scale $\leq 2$; (3) evaluable disease with or without measurable lesions; (4) disease progression after previous chemotherapies within 3 months before entry, with a maximum of three previous regimens, including neoadjuvant or adjuvant chemotherapy; and (5) adequate hematological, renal, and hepatic functions. The latter was defined as neutrophil $\geq 1,500 / \mathrm{mm}^{3}$, platelet $\geq 100,000 /$ $\mathrm{mm}^{3}$, serum creatinine $\leq 1.5 \mathrm{mg} / \mathrm{dL}$, total bilirubin $\leq 1.25$ (or 1.5) $\times$ upper limit of normal (ULN), and serum transaminases $\leq 2.5$ (or 5.0) $\times$ ULN in the absence (or presence) of liver metastasis. Patients were excluded if they had concurrent malignancy within the past 5 years (excluding basal cell carcinoma of the skin or cervical carcinoma in situ), symptomatic metastasis to brain, or uncontrolled significant comorbidity. The protocol was approved by the Institutional Review Board, and all the patients gave informed consent before enrollment.

\section{Treatment and dose adjustments}

Irinotecan was administered biweekly on days 1 and 15 . The starting dose was $150 \mathrm{mg} / \mathrm{m}^{2}$ infused intravenously for $2 \mathrm{~h}$. The $\mathrm{S}-1$ dose was $35 \mathrm{mg} / \mathrm{m}^{2}$ twice daily, which was administered for 14 consecutive days (days 1-14) followed by a 14-day resting period. S-1 dosage was calculated based on the body surface area, which differs from the Japanese guide [15]. The cycle was repeated every 4 weeks. Patients were premedicated with routine antiemetics.

If grade 2 neutropenia occurred on the day 15, irinotecan dose was reduced to $120 \mathrm{mg} / \mathrm{m}^{2}$. If grade $\geq 3$ neutropenia developed, irinotecan was skipped. For patients developing grade 4 neutropenia (or any grade febrile neutropenia), grade 4 thrombocytopenia, grade $\geq 3$ or recurrent grade 2 diarrhea or mucositis, grade $\geq 3$ lethargy, or grade $\geq 2$ hyperbilirubinemia, treatment was stopped until resolution and restarted with reduced dose of S-1 $\left(30 \mathrm{mg} / \mathrm{m}^{2}\right.$ followed by $\left.25 \mathrm{mg} / \mathrm{m}^{2}\right)$ and irinotecan $\left(120 \mathrm{mg} / \mathrm{m}^{2}\right.$ followed by $100 \mathrm{mg} / \mathrm{m}^{2}$ ). The resolution of hematological toxicity was defined as neutrophil $\geq 1,500 / \mathrm{mm}^{3}$ and platelets $\geq$ $75,000 / \mathrm{mm}^{3}$, respectively. In the event of severe toxicities, despite dose modifications, irinotecan was further reduced to $75 \mathrm{mg} / \mathrm{m}^{2}$.

Granulocyte colony-stimulating factor (G-CSF) was therapeutically indicated when patients developed grade 4 neutropenia, but prophylactic use of G-CSF was also allowed to prevent more severe neutropenia. Patients who required $>6$-week delay to recover from any toxicity other than alopecia and anemia or who required dose reduction due to toxicity above the planned modification step were withdrawn from the study. Chemotherapy was continued 
until there was disease progression, unacceptable toxicity, or the patient's withdrawal.

\section{Response and toxicity assessment}

Baseline evaluations included a complete medical history with physical examination, performance status, complete blood count (CBC), serum chemistries, tumor markers [carcinoembryonic antigen (CEA), CA19-9], urinalysis, and electrocardiography. A radiological examination of each lesion was carried out within 3 weeks before the treatment. Fiberoptic gastroduodenoscopy was planned to evaluate complete responders. Physical examination, CBC, and serum chemistries were planned at days 1 and 15 of the cycle. For tumor response evaluation, imaging studies were repeated every two cycles.

Treatment response was evaluated using spiral computed tomography according to the guidelines of the Response Evaluation Criteria in Solid Tumors Committee (RECIST, version 1.0) by independent radiologists. The response was assessed according to intention-to-treat (ITT) analysis. Patients were considered assessable for response when they had received a minimum of two cycles with at least one tumor measurement, or when they had clinical or radiologic evidence of early disease progression within first two cycles. Toxicities were evaluated and recorded as a grade according to the NCI-Common Toxicity Criteria (version 3.0).

\section{Statistical analysis}

The primary endpoint of the study was overall survival (OS), and the secondary endpoints were PFS, ORR, disease-control rate (DCR), and toxicity. OS was defined from the treatment start to death of any cause. PFS was defined from the treatment start to disease progression or death of any cause. We tested the hypothesis that mean survival time (MST) would improve by $75 \%$ compared with historical controls. The study was designed to have a $90 \%$ power to show an improvement in OS from 3.8 to 6.6 months with a $10 \%$ type I error, using one-sided testing and assuming exponential OS times. Planned patient accrual time was 18 months, and follow-up period was 6 months. A sample size of 34 patients was required [16]. Considering a $10 \%$ dropout rate, 38 patients were needed for this trial. Association between clinicopathological parameters was analyzed with Fisher's exact test. Timedependent variables were analyzed using the Kaplan-Meier method and compared using the log-rank test. Multivariate analysis was carried out using Cox's proportional hazards regression model. Exact $95 \%$ confidence interval (CI) was provided for proportions.

\section{Results}

Patient characteristics

From May 2007 to December 2008, a total of 38 patients were enrolled. Patient characteristics are summarized in Table 1. The median age was 57 years. Eighteen patients $(47 \%)$ received study agents as second-line treatment and remaining 20 patients $(53 \%)$ as third- or fourth-line treatment. Twenty-two patients $(58 \%)$ had prior gastrectomy; among whom, 17 (45\%) got curative-aim resection.

Table 1 Patient characteristics

\begin{tabular}{|c|c|}
\hline $\begin{array}{l}\text { Baseline clinicopathologic } \\
\text { features }\end{array}$ & $\begin{array}{l}\text { Patient } \\
\text { number }\end{array}$ \\
\hline Total enrolled & 38 \\
\hline Response evaluable patients & 35 \\
\hline Age, median (range) & $57(34-77)$ \\
\hline \multicolumn{2}{|l|}{$\operatorname{Sex}(\%)$} \\
\hline Male & $21(55)$ \\
\hline Female & $17(45)$ \\
\hline \multicolumn{2}{|l|}{ Performance status (ECOG) } \\
\hline 0 & $7(18)$ \\
\hline 1 & $28(74)$ \\
\hline 2 & $3(8)$ \\
\hline \multicolumn{2}{|l|}{ Previous chemotherapy $(\%)$} \\
\hline First line & $18(47)$ \\
\hline Second line & $13(34)$ \\
\hline Third line & $7(18)$ \\
\hline \multicolumn{2}{|l|}{ Histology (\%) } \\
\hline Well and moderately differentiated & $12(32)$ \\
\hline Poorly differentiated & $16(42)$ \\
\hline Signet ring cell & $9(24)$ \\
\hline Others & $1(3)$ \\
\hline \multicolumn{2}{|l|}{ Previous gastrectomy (\%) } \\
\hline None & $16(42)$ \\
\hline Curative & $17(45)$ \\
\hline Palliative & $5(13)$ \\
\hline \multicolumn{2}{|l|}{ Number of metastasis site } \\
\hline 1 & $11(29)$ \\
\hline 2 & $14(37)$ \\
\hline$\geq 3$ & $13(34)$ \\
\hline \multicolumn{2}{|l|}{ Disease site $(\%)$} \\
\hline Abdominal lymph node & $24(31)$ \\
\hline Peritoneum & $19(25)$ \\
\hline Liver & $9(12)$ \\
\hline Cervical lymph node & $6(8)$ \\
\hline Lung & $3(4)$ \\
\hline Others & $16(21)$ \\
\hline
\end{tabular}

ECOG Eastern Cooperative Oncology Group 
Abdominal lymph nodes were the main site of metastasis. Peritoneal seeding was noted in 19 (25\%) patients.

Previous chemotherapy histories of the patients are summarized in Table 2. There was a median of 23 (range 10-91) days from documenting disease progression of previous treatment to study treatment. Fourteen patients had progressive disease during chemotherapy-off period. Median 7.5 cycles (range 2-14) of first-line chemotherapy were administered, with a median relative dose intensity (RDI) of 0.97 (range 0.76-1.0). The ORR of first-line chemotherapy was $37 \%$. Twenty patients received secondline chemotherapy with a median cycle, and RDI were four (range 2-14) and 0.95 (range 0.64-1.0), respectively. The ORR of second-line chemotherapy was $20 \%$. Finally, seven patients received third-line chemotherapy for the median four cycles (range 2-6), with the median RDI of 0.86 (range 0.63-1.0) and 14\% ORRs. This implies that the patients enrolled received enough doses and cycles of previous chemotherapy.

\section{Treatment summary}

A total of 208 cycles were administered, with a median of four cycles (range 1-16) per patient. The median dose intensity of irinotecan and S-1 was $61 \mathrm{mg} / \mathrm{m}^{2} /$ week (range $27.8-71.0$ ) and $210 \mathrm{mg} / \mathrm{m}^{2} /$ week (range $91-245 \mathrm{mg} / \mathrm{m}^{2} /$ week), respectively, which corresponds to RDIs of 0.81 and 0.86 , respectively. All but nine patients had to delay next cycles with the median delay of 3 weeks (range 2-6). Ten patients were subjected to dose reduction due to toxicity; hematologic toxicity $(n=5)$, diarrhea $(n=3)$, and mucositis $(n=2)$, respectively. After PD on this regimen, ten patients (55\%) who received study treatment as second line were transferred to further chemotherapy: taxane monotherapy $(n=5)$ and oxaliplatin combination $(n=5)$.
Nine patients (44\%) as third- or fourth-line treatment were transferred to further chemotherapy: taxanes monotherapy $(n=4)$, oxaliplatin combination $(n=3)$, and other oral fluorouracils $(n=2)$.

\section{Efficacy}

With the median follow-up duration of 8.2 months (range 3.4-23.1), 33 patients had PD and $30(79 \%)$ died from cancer. The MST was 8.7 months (95\% CI 7.5-10.3) (Fig. 1a); 11.6 months (95\% CI 4.8-18.4) for the secondline treatment group and 7.6 months (95\% CI 3.9-8.8) for the third-line or more treatment groups. The one-year survival rate of all patients was $36 \%$ (42\% for the secondline group, $20 \%$ for the third-line group, and $14 \%$ for the fourth-line group).

The median PFS was 6.3 months (95\% CI 5.3-7.3) (Fig. 1b); 6.5 months (95\% CI 5.3-7.7) for the second-line group, and 5.6 months (95\% CI 3.9-7.3) for the third-line or more treatment groups. On multivariate analysis for survival, low serum albumin $(<3.5 \mathrm{mg} / \mathrm{dL})(\mathrm{OR}=6.43$, $P<0.001)$ was the most significant independent adverse prognosticators for OS (Table 3). Old age $(\geq 65)$ and prior exposure to capecitabine comprised other independent factors for poor survival. For PFS, liver metastasis $(\mathrm{OR}=5.74)$ and low serum albumin $(\mathrm{OR}=2.89)$ were the adverse factors.

Tumor response was assessable in all but three patients who had non-measurable lesions only. Objective responses were observed in 6 patients, and 19 had stable disease (SD). The ORR was $17 \%$ (95\% CI 4-30\%). Among the objective responders, four received the study agents as second-line treatment and the other two patients as thirdline treatment. Their median response duration was 4.5 months (range 2.0-12.0).
Table 2 Summary of prior chemotherapy regimens

$N A$ not assessable, $R D I$ relative dose intensity, 5-FU

5-fluorouracil

a Pemetrexed + cisplatin; capecitabine + doccetaxel

b 5 -FU + adriamycin $(n=2)$,

5-FU + cisplatin,

capecitabine + cisplatin,

vinflunine

\begin{tabular}{lccc}
\hline Treatment group & $\begin{array}{l}\text { Number of enrolled } \\
\text { patients }\end{array}$ & $\begin{array}{l}\text { Median cycles } \\
\text { (range) }\end{array}$ & $\begin{array}{l}\text { Median RDI } \\
\text { (range) }\end{array}$ \\
\hline First line & 38 & $7.5(2-14)$ & $0.97(0.76-1)$ \\
(Oral) 5-FU + cisplatin & 12 & $6(3-12)$ & $1.0(0.76-1)$ \\
Taxanes + 5-FU & 9 & $8(2-12)$ & $1.0(0.81-1)$ \\
Taxanes + cisplatin & 9 & $8(3-11)$ & $0.96(0.89-1)$ \\
(Oral) 5-FU + oxaliplatin & 6 & $8.5(6-14)$ & $1.0(0.89-1)$ \\
Others & a & NA & NA \\
Second line & 2 & $4(2-14)$ & $0.95(0.64-1)$ \\
Taxanes + 5-FU & 10 & $2(1-9)$ & $0.93(0.64-1)$ \\
(Oral) 5-FU + oxaliplatin & 5 & $9(2-14)$ & $0.95(0.76-1)$ \\
Others & b & NA & NA \\
Third line & 5 & $4(2-6)$ & $0.86(0.63-1)$ \\
(Oral) 5-FU + oxaliplatin & 4 & $3.5(2-6)$ & $0.82(0.63-1)$ \\
Taxanes & 3 & $4(4-6)$ & $0.86(0.73-1)$ \\
\hline
\end{tabular}


Fig. 1 Kaplan-Meier curves a progression-free survival, b overall survival
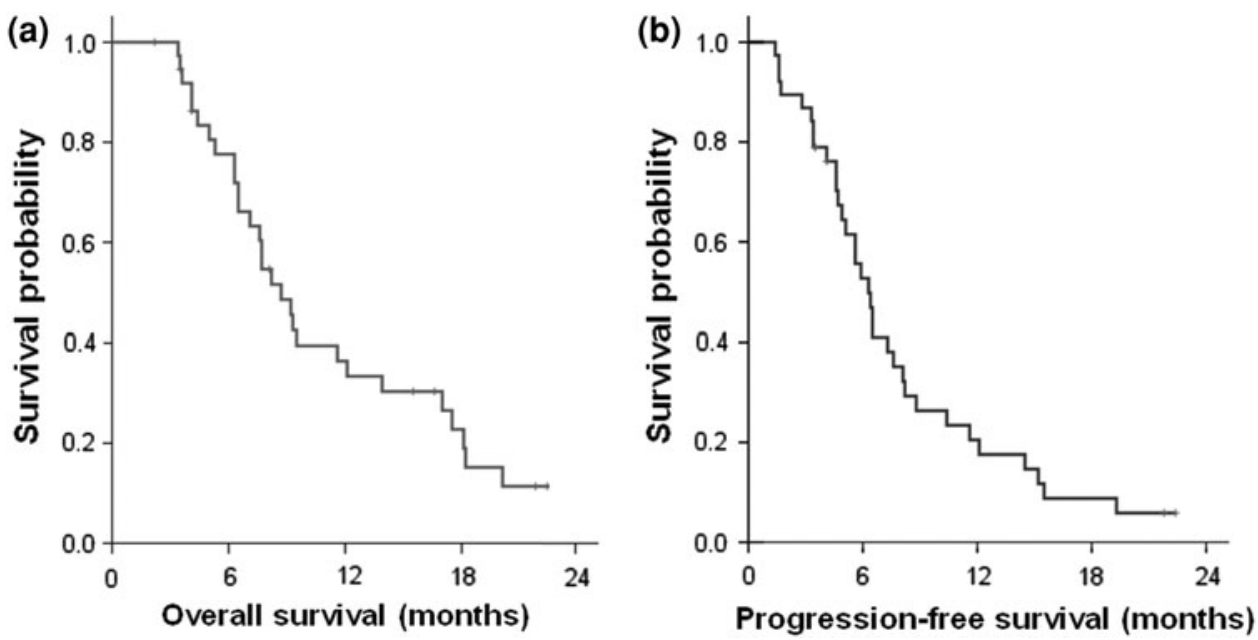

The DCR was $71 \%$ (95\% CI 56-86\%). The median duration of disease control was 7.3 months (range 2.0-22.4). We evaluated DCR separately by previous treatment. For the second-line treatment group, the DCR was $83 \%$ and the median duration was 8.1 months (range 3.3-19.3). For the third-line or more treatment groups, the DCR was $60 \%$, with the median duration of 5.7 months (range 3.5-22.4).

\section{Toxicity}

The toxicity profile is summarized in Table 4. There was no treatment-related mortality. The most common grade $3 / 4$ hematologic toxicity was neutropenia, which was found in $26 \%$ of the patients, and three patients $(8 \%)$ suffered febrile neutropenia. Grade 3/4 leukopenia and anemia were also observed in 27 and $19 \%$ of the patients, respectively. Twenty-three patients required G-CSF support, but none of them suffered febrile neutropenia. The median frequency of G-CSF administration was 2 (range 1-10). The most common grade 3/4 non-hematological toxic effects were diarrhea $(18 \%)$, mucositis $(8 \%)$, and hyperbilirubinemia $(6 \%)$.

\section{Discussion}

Whether the benefit of chemotherapy will continue with disease progression during or after first-line treatment is still unclear [11]. There are few randomized trials investigating the efficacy and safety of salvage chemotherapy, and most phase II trials are from Japan, Korea, and Italy, where the practice of offering second-line chemotherapy is common [1]. But it is consistent findings from pooled analysis that responders to second-line chemotherapy survive longer compared with non-responders, and symptomatic benefit may be obtained from the second-line therapy [17]. Old agents have been rapidly replaced by newer drugs including taxanes, irinotecan, and oxaliplatin, which prove to be more effective and better tolerated in gastric cancer. Therefore, we designed this study of combined S-1 and irinotecan assuming that newer drugs are a rational option for salvage treatment as it is for first-line treatments.

The first point to consider is the treatment schedule. Many schedules and combination partners have been designed for irinotecan. For second-line treatment, irinotecan has been tried as monotherapy-weekly or biweekly - as well as in combination with 5-FU, capecitabine, cisplatin, or mitomycin [8-10, 18, 19]. These studies demonstrated ORR of $15-30 \%$ and OS of 5-8 months. However, IRIS has been tried only on previously untreated patients. Two Japanese trials adopted biweekly irinotecan, but its dosage differed from each other. Uedo et al. tried $80 \mathrm{mg} / \mathrm{m}^{2}$ (40 mg/m²/week) and reported ORR of $48 \%$, while Komatsu et al., derived from phase I study by themselves, adopted $125 \mathrm{mg} / \mathrm{m}^{2}\left(62.5 \mathrm{mg} / \mathrm{m}^{2} /\right.$ week $)$ to obtain better ORR of $54 \%$ [20, 21]. A Korean study favored triweekly schedule with $150 \mathrm{mg} / \mathrm{m}^{2}\left(50 \mathrm{mg} / \mathrm{m}^{2} /\right.$ week) irinotecan with ORR of $49 \%$ [22]. Despite some various ranges of $\mathrm{S}-1$ dose among the studies, we thought that these findings mainly implied dose-response relationship of irinotecan might exist. On designing this study, we knew about the phase I study that biweekly $150 \mathrm{mg} / \mathrm{m}^{2}$ irinotecan did not bring about dose-limiting toxicity [23]. Additionally, a recent phase III study (GC0301/TOP-002) comparing IRIS with S-1 monotherapy failed to demonstrate the superiority of the combination therapy [23]. We assumed that this failure might be due to the low dose intensity of irinotecan ( $32 \mathrm{mg} / \mathrm{m}^{2} /$ week), which is only half the currently accepted dose. Therefore, we thought that $150 \mathrm{mg} / \mathrm{m}^{2}$ irinotecan could be tolerable, even in previously treated population, if their general condition is permissible.

One critical point considered in designing second-line treatment is the homogeneity of study population. 


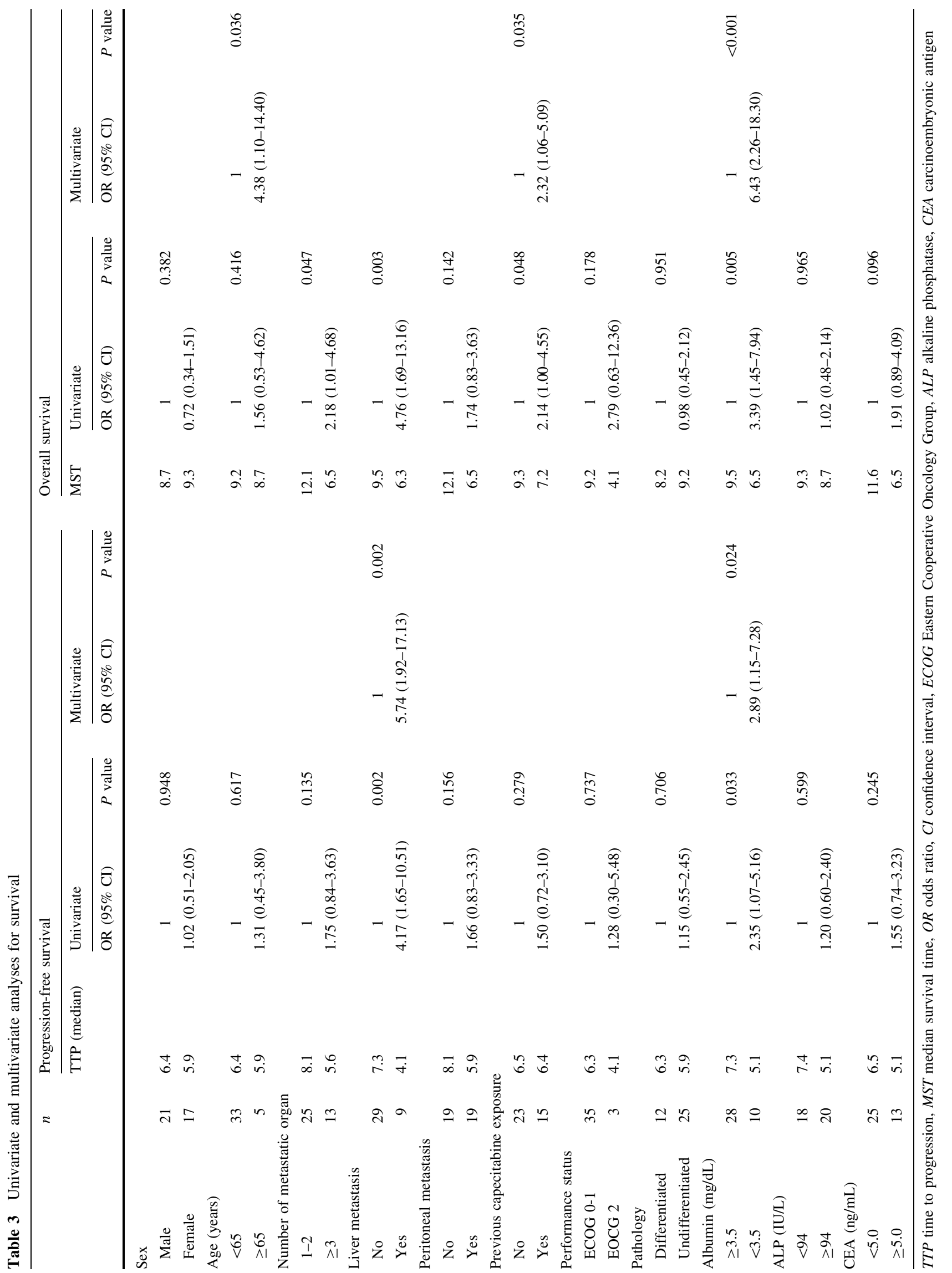


Table 4 Toxicity profile

\begin{tabular}{|c|c|c|c|c|c|c|}
\hline & \multicolumn{4}{|c|}{ Number of patients (\%) } & \multirow{2}{*}{$\begin{array}{l}\text { Toxicity of } \\
\text { all grades }(\%)\end{array}$} & \multirow{2}{*}{$\begin{array}{l}\text { Toxicity of } \\
\text { grades 3-4 (\%) }\end{array}$} \\
\hline & Grade 1 & Grade 2 & Grade 3 & Grade 4 & & \\
\hline \multicolumn{7}{|l|}{ Hematologic toxicity } \\
\hline Neutropenia & $1(3)$ & $8(21)$ & $8(21)$ & $2(5)$ & 50 & 26 \\
\hline Anemia & - & $21(55)$ & $6(16)$ & $1(3)$ & 74 & 19 \\
\hline Leukopenia & $9(24)$ & $6(16)$ & $9(24)$ & $1(3)$ & 67 & 27 \\
\hline Thrombocytopenia & - & $1(3)$ & - & $1(3)$ & 6 & 3 \\
\hline \multicolumn{7}{|l|}{ Non-hematologic toxicity } \\
\hline Nausea/vomiting & $4(10)$ & $12(32)$ & $1(3)$ & - & 46 & 3 \\
\hline Mucositis & $1(3)$ & $1(3)$ & $3(8)$ & - & 8 & 8 \\
\hline Diarrhea & $10(26)$ & $15(39)$ & $5(13)$ & $2(5)$ & 83 & 18 \\
\hline Hand-food syndrome & $1(3)$ & $1(3)$ & $1(3)$ & - & 9 & 3 \\
\hline Constipation & $2(5)$ & $2(5)$ & - & - & 10 & - \\
\hline Peripheral neuropathy & $1(3)$ & $3(8)$ & - & - & 11 & - \\
\hline Elevated creatinine & $3(8)$ & - & - & - & 8 & - \\
\hline Elevated liver enzyme & $6(16)$ & $2(5)$ & $1(3)$ & - & 24 & 3 \\
\hline Hyperbilirubinemia & $3(8)$ & $1(3)$ & $1(3)$ & $1(3)$ & 19 & 6 \\
\hline
\end{tabular}

Studies of a number of tumor types, including gastric cancer, have shown that the progression-free interval after first-line treatment correlates with a benefit from second-line treatment [1, 24]. Therefore, we think that our study offers clinical insight into high-risk patients that progressed during or within 3 months of prior chemotherapy.

The other point is the study aim. We set up the primary endpoint as median survival, not the more traditional endpoints, response rate. Although the activity of salvage chemotherapy can be seen in terms of response rate, there are substantial variations in findings (4-38\%), which result from small patient numbers and heterogenous populations in terms of various different previous regimens [1]. Therefore, the interpretation of trials testing second-line treatment by ORR is difficult. Moreover, in gastric cancer, tumor response poorly correlates with survival and a study demonstrates that the response of a primary gastric mass poorly correlates with a metastatic lesion [25]. Patients often progresses in non-measurable disease, most of which cannot be measured by conventional imaging [26]. Therefore, we assumed that OS rather reflects the benefit from chemotherapy especially in salvage setting, and the more important is selecting subgroup most likely to benefit from the chemotherapy. Of course, we admit that OS is not an optimal endpoint, either; it is influenced by many other factors including subsequent therapies; about half of patients received subsequent therapy after the current treatment. Other endpoints such as better symptom control, quality of life, or improvement of performance might be further investigated.
A pooled analysis of three randomized trials of fluorouracil-based chemotherapy in AGC reported that the MST of second-line chemotherapy was 5.6 months [27]. The survival duration from PD to death after first-line treatment in a recent Korean study reached 6.6 months, longer than 3-5 months in Western studies in which second-line or subsequent salvage chemotherapies were not usually offered [2]. Our study included heavily treated patients of third- or fourth-line treatment, and we assumed that the historical control of 3.8 months to be a reasonable reference. We designed to show a $75 \%$ increase in OS to 6.6 months, which we thought was somewhat high considering prior exposure to chemotherapy. Nevertheless, we believe that this survival parameter is currently the minimum range for the acceptance of irinotecan-containing regimens aiming at salvage treatment in AGC [2, 8-10, 19]. We demonstrated that the MST reached 8.7 months. Although direct comparison with previous phase II studies is not amenable, our documented survival profiles are in the upper level of other irinotecan-based combinations $[18,28]$. This could be partly explained that the patients enrolled seem to be highly selected; they appeared to have chemosensitive tumor on considering their previous chemotherapy history and dose intensity and to have low tumor burden (peritoneal metastasis 25\%, liver metastasis $12 \%$ ) when compared with previous studies of salvage chemotherapy [18, 28]. Another factor could be a high proportion of good performance status patients, and general condition was maintained throughout the treatment course, which enabled many patients to transfer to subsequent chemotherapy protocols. 
Because deteriorating performance status and nutritional status are common in gastric cancer patients, to select proper candidates who may benefit from salvage treatment is important. We allowed previous exposure to capecitabine, but none of these patients responded. The group previously exposed to capecitabine had a significantly shorter OS than capecitabine-naïve group (relative risk $=3.1, P=0.012$ ). In breast cancer, $S-1$ had very limited activity in capecitabine-pretreated patients who had already been exposed to anthracycline and taxanes, which implies that S-1 clinically exhibited cross-resistance to capecitabine [29]. Our data provide additional evidence-although indirect- that capecitabine resistance may adversely influence S-1 efficacy and should be a consideration for eligibility in future clinical trials. Another independent prognostic factor is hypoalbuminemia. Performance status was not a significant factor for survival due to the small patient number of ECOG 2. Instead, serum albumin level as a predictive marker indicated that patients of hypoalbuminemia $(\leq 3.5 \mathrm{~g} / \mathrm{dL})$ had 2.5 times higher risk of death. Hypoalbuminemia is a consistent prognostic factor in gastric cancer patients in palliative setting [30, 31]. Although many confounding factors-comorbidity, anorexia/malnutrition, dysregulation of cytokines, inflammation-associate between albumin and survival, we suggest that serum albumin could be used in clinical trials to better define the baseline risk in AGC. We believe that visceral protein depletion, inflammatory response markers, and nutritional strategy should be further studied along with cancerspecific therapy.

The incidence of neutropenia and diarrhea seemed higher compared with other phase II studies of S-1 plus irinotecan, which is comparable with capecitabine plus irinotecan chemotherapy [19, 21-23]. The high-dose intensity of irinotecan may, at least partly, contribute the relative increase in toxicity. However, the development of pharmacogenetic markers of irinotecan and S-1 can help us determine, which patients might avoid excessive toxicity while benefit from second-line chemotherapy.

To summarize, our first phase II study demonstrated that biweekly IRIS regimen was well tolerated and showed encouraging survival profile. These findings reaffirm that irinotecan-based therapy has use as salvage treatment for advanced gastric cancer. However, given the high incidence of neutropenia and gastrointestinal toxicity, proper patient selection may be warranted; relatively younger patients $(<65)$ with normal serum albumin level may the best candidates for the treatment, while patients with liver metastasis compromising liver functions or who have prior capecitabine exposure should be an exclusion criteria for the application of this regimen.
Conflict of interest No conflict of interest exists in the submission of this article.

Open Access This article is distributed under the terms of the Creative Commons Attribution Noncommercial License which permits any noncommercial use, distribution, and reproduction in any medium, provided the original author(s) and source are credited.

\section{References}

1. Wesolowski R, Lee C, Kim R (2009) Is there a role for secondline chemotherapy in advanced gastric cancer? Lancet Oncol 10:903-912

2. Moon YW, Rha SY, Jeung HC, Kim C, Hong MH, Chang H, Roh JK, Noh SH, Kim BS, Chung HC (2010) Outcomes of multiple salvage chemotherapy for advanced gastric cancer: implications for clinical practice and trial design. Cancer Chemother Pharmacol 66:797-805

3. Lacave AJ, Izarzugaza I, Antón Aparicio LM, Valle Pereda M, Gracia Marco JM, Buesa JM (1983) Phase II clinical trial of cis-dichlorodiammineplatinum in gastric cancer. Am $\mathrm{J}$ Clin Oncol 6:35-38

4. Lacave AJ, Wils J, Diaz-Rubio E, Clavel M, Planting A, Bleiberg H, Duez N, Dalesio O (1985) cis-Platinum as second-line chemotherapy in advanced gastric adenocarcinoma. A phase II study of the EORTC gastrointestinal tract cancer cooperative group. Eur J Cancer 21:1321-1324

5. Ohtsu A, Yoshida S, Saito D, Shimada Y, Miyamoto K, Fujii T, Yoshino M, Yoshimori M (1991) An early phase II study of 5-fluorouracil combined with cisplatinum as a second line chemotherapy against metastatic gastric cancer. Jpn J Clin Oncol $21: 120-124$

6. Cascinu S, Graziano F, Cardarelli N, Marcellini M, Giordani P, Menichetti ET, Catalano G (1998) Phase II study of paclitaxel in pretreated advanced gastric cancer. Anticancer Drugs 9:307-310

7. Stathopoulos GP, Rigatos SK, Fountzilas G, Polyzos A, Stathopoulos JG (2002) Paclitaxel and carboplatin in pretreated advanced gastric cancer: a phase II study. Oncol Rep 9:89-92

8. Shimada S, Yagi Y, Kuramoto M, Aoki N, Ogawa M (2003) Second-line chemotherapy with combined irinotecan and lowdose cisplatin for patients with metastatic gastric carcinoma resistant to 5-fluorouracil. Oncol Rep 10:687-691

9. Chun JH, Kim HK, Lee JS, Choi JY, Lee HG, Yoon SM, Choi IJ, Ryu KW, Kim YW, Bae JM (2004) Weekly irinotecan in patients with metastatic gastric cancer failing cisplatin-based chemotherapy. Jpn J Clin Oncol 34:8-13

10. Kanat O, Evrensel T, Manavoglu O, Demiray M, Kurt E, Gonullu G, Kiyici M, Arslan M (2003) Single-agent irinotecan as secondline treatment for advanced gastric cancer. Tumori 89:405-407

11. Thuss-Patience PC, Kretzschmar A, Deist T, Hinke A, Bichev D, Lebedinzew B, Schumacher G, Gebauer B, Maier V, Reichardt P (2009) Irinotecan versus best supportive care (BSC) as secondline therapy in gastric cancer: a randomized phase III study of the Arbeitsgemeinschaft Internistische Onkologie (AIO). J Clin Oncol 27(15S):abstract 4540

12. Wagner AD, Grothe W, Haerting J, Kleber G, Grothey A, Fleig WE (2006) Chemotherapy in advanced gastric cancer: a systematic review and meta-analysis based on aggregate data. J Clin Oncol 24:2903-2909

13. Pavillard V, Formento P, Rostagno P, Formento JL, Fischel JL, Francoual M, Etienne MC, Milano G (1998) Combination of irinotecan (CPT11) and 5-fluorouracil with an analysis of cellular determinants of drug activity. Biochem Pharmacol 56:13151322 
14. Fukushima M (2006) Antitumor activity and functions of S-1, a new oral tegafur-based formulation. Gan To Kagaku Ryoho 33:S19-S26

15. Jeung HC, Rha SY, Kim HK, Lim HY, Kim S, Kim SY, Gong SJ, Park CH, Ahn JB, Noh SH, Chung HC (2007) Multi-institutional phase II study of S-1 monotherapy in advanced gastric cancer with pharmacokinetic and pharmacogenomic evaluations. Oncologist 12:543-554

16. Lawless J (1982) Statistical models and methods for lifetime data (Wiley series in probability \& mathematical statistics). Wiley, Hoboken

17. Wilson D, Hiller L, Geh JI (2005) Review of second-line chemotherapy for advanced gastric adenocarcinoma. Clin Oncol 17:81-90

18. Giuliani F, Molica S, Maiello E, Battaglia C, Gebbia V, Di Biscelie M, Vinciarelli G, Gebbia N, Colucci G (2005) Irinotecan (CPT-11) and mitomycin-C(MMC) as second-line therapy in advanced gastric cancer. Am J Clin Oncol 28:581-585

19. Leary A, Assersohn L, Cunningham D, Norman AR, Chong G, Brown G, Ross PJ, Costello C, Higgins L, Oates J (2009) A phase II trial evaluating capecitabine and irinotecan as second-line treatment in patients with oesophago-gastric cancer who have progressed on, or within 3 months of platinum-based chemotherapy. Cancer Chemother Pharmacol 64:455-462

20. Uedo N, Narahara H, Ishihara R, Takiuchi H, Goto M, Fujitani K, Hirao $M$, Tsujinaka $T$, Imano $M$, Furukawa $H$, Tsukuma $H$, Taguchi T (2007) Phase II study of a combination of irinotecan and S-1 in patients with advanced gastric cancer (OGSG0002). Oncology 73:65-71

21. Komatsu Y, Yuki S, Fuse N, Kato T, Miyagishima T, Kudo M, Kunieda Y, Tateyama M, Wakahama O, Meguro T, Sakata Y, Asaka M (2010) Phase 1/2 clinical study of irinotecan and oral S-1 (IRIS) in patients with advanced gastric cancer. Adv Ther 27:483-492

22. Kang HJ, Chho SH, Oh SJ, Yang SH, Lee MH, Song EK, Chung HC, Na II, Ryoo BY (2009) Phase II study of S-1 and irinotecan combination chemotherapy as a first-line therapy for patients with advanced gastric cancer. Korean cancer study group protocol ST05-02. Asia Pac J Clin Oncol 5:46-54

23. Imamura $H$, Iishi $H$, Tsuburaya $A$, Hatake $K$, Imamoto $H$, Esaki T, Kato M, Furukawa H, Hamada C, Sakata Y (2008) Randomized phase III study of irinotecan plus S-1 (IRIS) versus S-1 alone as first-line treatment for advanced gastric cancer (GC0301/TOP002). In: Gastrointestinal cancers symposium, abstr 5

24. Stahl M, Müller C, Köster W, Wilke H (2005) Second-line chemotherapy of advanced disseminated gastric cancer after cisplatin, infusional 5-fluorouracil, folinic acid (PLF): benefit dependent on progression-free interval after first-line therapy. Onkologie 28:499-502

25. Yoshida S, Miyata Y, Ohtsu A, Boku N, Shirao K, Shimada Y (2000) Significance of and problems in adopting response evaluation criteria in solid tumor RECIST for assessing anticancer effects of advanced gastric cancer. Gastric Cancer 3:128-133

26. Yoo CH, Noh SH, Shin DW, Choi SH, Min JS (2000) Recurrence following curative resection for gastric carcinoma. Br J Surg 87:236-242

27. Chau I, Norman AR, Ross PJ, Waters JS, Oates J, Cunningham D (2004) Multivariate prognostic factor analysis and second line treatment in locally advanced and metastatic oesophago-gastric cancer-pooled analysis of 1,080 patients from three multicentre randomised controlled trials using individual patient data. In: Gastrointestinal cancers symposium 2004, abst 5

28. Sym SJ, Chang HM, Kang HJ, Lee SS, Ryu MH, Lee JL, Kim TW, Yook JH, Oh ST, Kim BS, Kang YK (2008) A phase II study of irinotecan and docetaxel combination chemotherapy for patients with previously treated metastatic or recurrent advanced gastric cancer. Cancer Chemother Phamacol 63:1-8

29. Ito Y, Osaki Y, Tokudome N, Sugihara T, Takahashi S, Iwase T, Hatake K (2009) Efficacy of S-1 in heavily pretreated patients with metastatic breast cancer: cross-resistance to capecitabine. Breast Cancer 16:126-131

30. Alici S, Kaya S, Izmirli M, Tuncer I, Doğan E, Ozbek H, Sayarlioglu H (2006) Analysis of survival factors in patients with advanced-stage gastric adenocarcinoma. Med Sci Monit 12:CR221-CR229

31. Lee J, Lim T, Uhm JE, Park KW, Park SH, Lee SC, Park JO, Park YS, Lim HY, Sohn TS, Noh JH, Heo JS, Park CK, Kim S, Kang WK (2007) Prognostic model to predict survival following firstline chemotherapy in patients with metastatic gastric adenocarcinoma. Ann Oncol 18:886-891 